\section{Prostatektomie: Wer profitiert am meisten?}

\author{
Bei welchen Patienten mit lokal begrenztem Prostatakarzinom kann eine \\ radikale Prostatektomie von größtem Nutzen sein? Einer schwedischen \\ Modellrechnung zufolge sind die Ergebnisse eindeutig.
}

\begin{abstract}
A nhand der Daten der Studie SPCG-4 (Scandinavian Prostate Cancer Group 4 Trial) haben skandinavische und US-amerikanische Wissenschaftler in einer Modellrechnung versucht zu klären, welche Männer mit einem lokal begrenzten Prostatakarzinom am meisten von einer radikalen Prostatektomie (RP) profitieren. Insgesamt 695 Patienten mit einem T1- oder T2-Tumor hatten an der Studie teilgenommen.

Im Studienzeitraum von zwölf Jahren starben 137 Männer der Interventionsgruppe und 156 Männer mit WatchfulWaiting-Strategie. Ein Prostatakarzinom als Todesursache wurde bei 47 Männern in der RP-Gruppe und bei 68 Männern in der anderen Gruppe dokumentiert.

Die Modellrechnung ergab, dass bei jüngeren Patienten mit einer eher aggressiven Erkrankung das Risiko, an den Fol-
\end{abstract}

gen eines Prostatakarzinoms zu sterben, stärker durch eine RP verringert werden kann als bei älteren Männern mit einem weniger aggressiven Tumor. Ein Beispiel: Bei einem 60-Jährigen mit einem Gleason-Score von 7 und einem T2-Tumor lässt sich das Risiko, nach zehn Jahren an Prostatakrebs zu sterben, durch eine RP statt einer Watchful-Waiting-Strategie von $24 \%$ auf $9 \%$ senken. Bei einem 70-Jährigen dagegen mit einem Gleason-Score von 6 und einem T1-Tumor gelingt nur eine Reduktion von $4 \%$ auf $3 \%$.

Insgesamt betrachtet bestätigten die Ergebnisse der Modellrechnung die gängige Praxis - etwa, dass bei jüngeren $\mathrm{Pa}$ tienten mit einem Gleason-Score von 8 eine RP sinnvoll ist und bei Patienten mit einem Gleason-Score von 6 und einem T1-Tumor Beobachten ausreicht. Neu sei die Erkenntnis, das bei Männer mit einem
Gleason-Score von 6 und einem T2-Tumor sowie Männern mit einem GleasonScore von 7 und einem T1-Tumor der Nutzen einer RP im Vergleich zum Watchful-Waiting nicht so eindeutig ist.

Fazit: Der schwedischen Modellrechnung zufolge profitieren vor allem Männer mit einem Tumor im Stadium T2 und einem Gleason-Score 7 oder 8 von einer radikalen Prostatektomie. Kaum zu rechtfertigen ist die Operation dagegen der Interpretation der Studienergebnisse zufolge bei Männern mit Gleason-Score 6 und Tumorstadium 1 sowie bei Männern, die deutlich älter als 70 Jahre sind. Nach Ansicht der Autoren handelt es sich um eine Ermessensentscheidung, für die die Wünsche des Patienten - etwa im Zusammenhang mit den zu erwartenden negativen Folgen des Eingriffes - und klinische Parameter wie Komorbiditäten berücksichtigt werden sollten.

Peter Leiner

Vickers A et al. Individualized Estimation of the Benefit of Radical Prostatectomy from the Scandinavian Prostate Cancer Group Randomized Trial. Eur Urol 2012 ; 62: 204-9

\section{Harninkontinenz-Therapie: Auf die Nebenwirkungen kommt es an}

Die einzelnen Präparate zur Behandlung der Dranginkontinenz unterscheiden sich in ihrer Wirksamkeit nur geringfügig, so das Ergebnis einer großen Metaanalyse. Behandelnde Ärzte sollten die Therapie daher vor allem am Nebenwirkungsprofil ausrichten.

W as ist die beste medikamentöse Therapie der weiblichen Dranginkontinenz? Um diese Frage zu beantworten, haben US-amerikanische Urologen in einer Metaanalyse 94 placebokontrollierte Studien begutachtet. Besonderen Wert legten sie dabei auf die Kontinenzraten sowie auf die Verträglichkeit der Therapien.

Insgesamt wurden $13 \%$ der mit Fesoterodin behandelten Frauen wieder kontinent, 11,4\% waren es jeweils mit Oxybutynin und Trospiumchlorid, 10,7\% mit Solifenacin und $8,5 \%$ mit Tolterodin allerdings sind diese Zahlen nicht direkt vergleichbar, da in den Studien unterschiedliche Patientengruppen behandelt wurden. In 21 Studien wurden jedoch auch Medikamente direkt verglichen. Dabei war Fesoterodin etwas effektiver als Tolterodin bei den Kontinenzraten und bei der Symptomlinderung. Allerdings kam es unter Fesoterodin auch häufiger zu Therapieabbrüchen als mit Tolterodin oder Oxybutynin. In den Vergleichsstudien war zudem Trospiumchlorid wirksamer als Oxybutynin, und mit Solifenacin war die Kontinenzrate höher als mit Tolterodin.

Über alle Studien gemittelt waren die Abbruchraten aufgrund von Nebenwirkungen mit Oxybutynin am höchsten (6,3\%), gefolgt von Fesoterodin $(3,1 \%)$, Trospiumchlorid $(1,8 \%)$ und Solifenacin
(1,3\%). Die häufigste Nebenwirkung war Mundtrockenheit, dazu kam es am häufigsten bei einer Therapie mit Oxybutynin.

Fazit: Die Medikamente waren alle deutlich wirksamer als Placebo, die Unterschiede bei der Wirksamkeit der einzelnen Präparate war aber eher gering, sodass für eine Therapieentscheidung vor allem das Nebenwirkungsprofil relevant ist. Die Autoren geben zu bedenken, dass die meisten der kontrollierten Studien nur zwei bis drei Monate dauerten und es insgesamt wenig Angaben gab, ob und wie stark sich die Lebensqualität der Frauen durch die Therapie verbesserte. Offene Studien hätten deutlich höhere Abbruchraten gezeigt als placebokontrollierte Studien, was daran liegen könnte, dass die im Praxisalltag übliche Polypharmazie die Nebenwirkungen noch verstärkt.

Thomas Müller

Shamliyan T et al. Systematic Review: Benefits and Harms of Pharmacologic Treatment for Urinary Incontinence in Women. Ann Intern Med 2012; 156: 861-74 